\title{
DESIGN AND FABRICATION OF A MICRO COULTER COUNTER WITH THIN FILM ELECTRODES
}

\author{
Siyang Zheng, Yu-Chong Tai \\ Electrical Engineering, California Institute of Technology \\ Pasadena, California 91125, USA
}

\begin{abstract}
We report a new approach for micro Coulter counter with thin film electrodes. Traditional Coulter counter measures DC resistance change to sense particles passing through an aperture between two flow chambers. One of the biggest challenges to miniaturize the device is to overcome the large resistance encountered. We propose a new approach to use thin film metal electrodes in micron size range for sensing. Resolution can be improved and it's easy for system integration. A device based on this approach was built and tested.
\end{abstract}

Keyword: Coulter counter, thin film metal electrodes, impedance, particle sensing

\section{INTRODUCTION}

Coulter counter is a well established method for particle counting and size measurement since 1950s. Since its introduction, Coulter counter has gradually replaced manual blood count with hemacytometer and becomes the standard instrument for clinic blood count. At the same time, its theory and technology have developed from DC resistance sensing to $\mathrm{AC}$ impedance sensing. With the later technology in combination with other technologies, such as light diffraction and immuno-staining, leukocyte differential are performed in clinic lab on daily basis.

Over the past decade or so, people from different research groups have attempted to miniaturize the Coulter counter with micro fabrication technologies. To solve the problem of large impedance at low frequency which is intrinsic to micro electrodes, several approaches have been attempted. $\mathrm{AC}$ impedance sensing has been used by several groups with different levels of success [1-3]. For DC resistive sensing, using gold pins [4] and large metal pads [5] as electrodes has been demonstrated. Recently, a novel salt bridge material was patterned as electrodes and combined with $\mathrm{Ag} / \mathrm{AgCl}$ electrodes to achieve low resistance [6]. Here we propose an approach that uses four electrodes. The external pair of electrodes has large surface area. It is used for excitation. We use both DC and low excitation frequency of $100 \mathrm{~Hz}$. The later one solves several problems associated with DC resistive sensing. The cycling of oxidation and reduction on electrode surface prevents significant loss of metal electrodes. It also speeds up the mass transfer of redox species near electrodes by not letting them to enter diffusion limited regime. Finally possible bubble generation by water hydrolysis can be minimized. The internal pair of electrodes has small surface area for sensing. It is very close to the aperture. The closeness of the two electrodes improves sensitivity and reduces false count by limiting the average number of particles between the two electrodes.

\section{PRINCIPLE}

The device consists of two flow chambers separated by an aperture (figure 1). Particles have different electrical properties than the electrolyte solvent Particle flowing through the aperture will induce an electrical resistance change as the solvent volume at the aperture is displaced by the particle. This change is measured by two electrodes placed in the two chambers respectively. The integration of the signal change over time is proportional to particle volume.

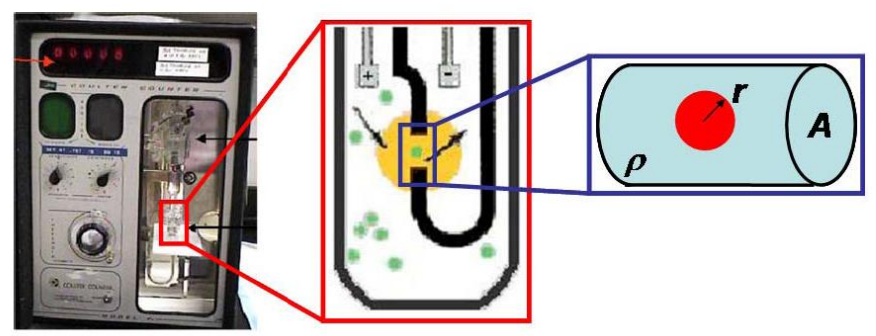

Figure 1: Principle of Coulter counter. The left picture is an early commercial instrument. The middle picture illustrates two chambers are separated by an aperture. The right picture shows a simplified model for the aperture part. Particle solution is forced to flow from one chamber to the other. The resistance change is measured by two electrodes.

Miniaturization can potentially improve device performance. The resistance change can be increased. Assuming uniform electrical field in sensing zone, spherical particle, cylindrical 
aperture and ignorable electrical resistance inside chambers from aperture to electrod, the resistance change $\Delta R$ is

$$
\Delta R=2 \rho\left\{\frac{\arctan \left(r / \sqrt{R^{2}-r^{2}}\right)}{\pi \sqrt{R^{2}-r^{2}}}-\frac{r}{\pi R^{2}}\right\}
$$

with $r$ represents the radius of the particle, $R$ for the radius of the aperture and $\rho$ for the resistivity of medium. As shown in figure 2, shrinking the aperture dimension greatly increases the resistance change, which is proportional to the signal been sensed.

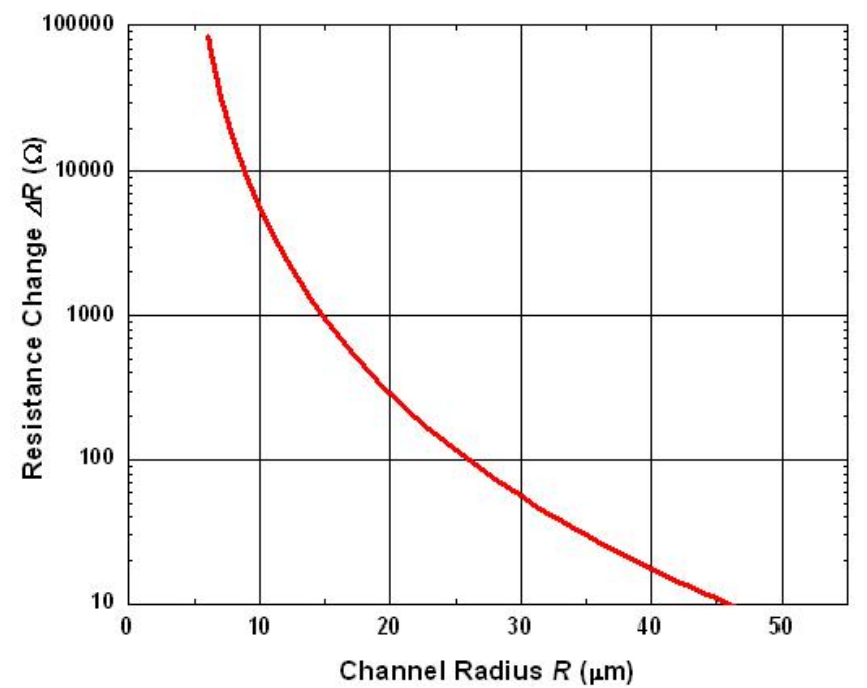

Figure 2: The relation between resistance change $\Delta R$ and aperture radius $R$, assuming $r$ is $5 \mu m$ and $\rho$ is $0.83 \Omega m$.

Another benefit of miniaturization is reducing the error rate caused by coincidence effect. The coincidence effect, which means two or more particles flow through aperture at the same time, can be modeled by Poisson process. The relationship of true count $N$ and observed count $n$ is:

$$
n=\frac{V}{\Delta V}\left(1-e^{-\frac{\Delta V}{V} N}\right)
$$

with $V$ for sample volume and $\Delta V$ for the aperture volume. Reducing the dimension of the sensing zone improves the error rate greatly as shown in figure 3 .

\section{DESIGN AND FABRICATION}

A major problem for scaling down the electrode size to micron range is the significant double layer effect on micro electrodes. The electrode-electrolyte interface can be modeled as a double lay er capacitor in parallel with a leaking resistor for the Faraday process. For DC or low frequency $\mathrm{AC}$, the double layer capacitor is effectively open circuit. On the other hand, the electrode surface resistance is inversely proportional to its area. The resistance between two micro electrodes is measured in Giga Ohm range and is dominated by their surface resistance. This makes the small resistance change induced by particle flowing through aperture very difficult to sense.

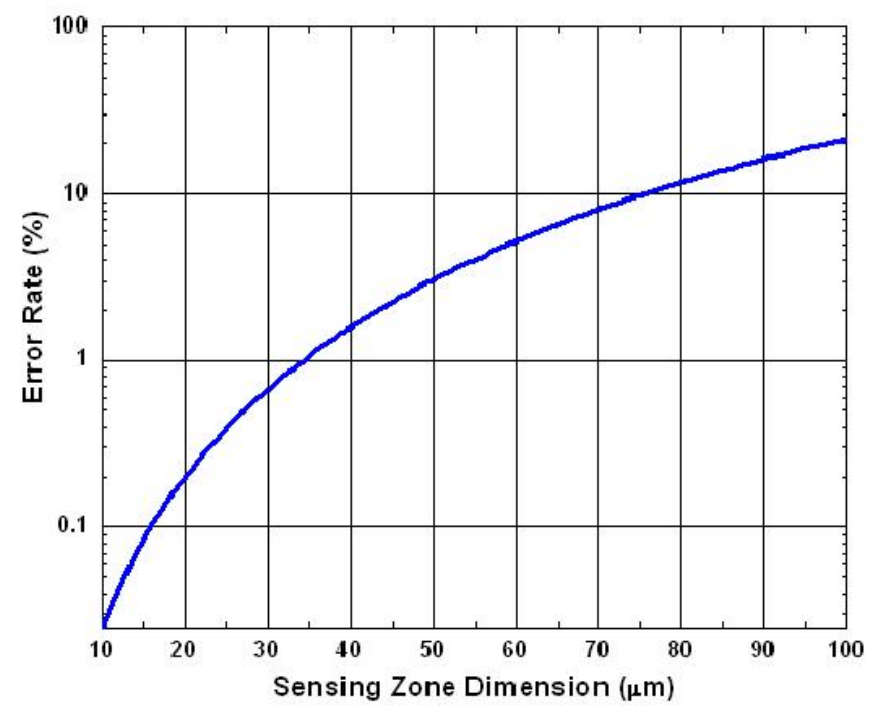

Figure 3: The relation between error rate and sensing zone dimension, assuming cubic sensing zone, sample volume $500 \mathrm{ul}$ and true count 250,000. The error rate is defined as $(n-N) / N \times 100 \%$.

To solve the problem, we propose using electrodes with large surface area, so that surface resistance is small enough to pump in micro Ampere range constant current. Near the aperture, sensing is achieved by small electrodes backed by high impedance buffer amplifier as input stage (figure 4), which withdraw neglectable amount of current. Thus introducing the sensing electrodes does not change the voltages at the sensing points. By doing this, we measure the true voltage difference across the aperture caused by electrolyte and particle without the influence of the double layer effect of electrodes. The small surface area of the sensing electrodes ensures superior special resolution of the sensing. We have a total of four sensing electrodes for added reliability and possible sensing of particle velocity.

The width of the aperture is designed to be $15 \mu \mathrm{m}$. The width of the sensing electrode is $20 \mu \mathrm{m}$. The channel height is $15 \mu \mathrm{m}$. We also include a filter region before the sensing zone to prevent any particle with size larger than the aperture to enter.

To fabricate the device, thin film Pt electrodes are evaporated onto soda-lime glass wafer with $\mathrm{Ti}$ used as adhesion layer and patterned by lift-off process. To increase the double layer capacitance and reduce the resistance, $\mathrm{Pt}$ 
black is electroplated onto the electrode surface. It has been shown that this can reduce AC impedance by order of two to three [7]. The mold for the channels is prepared by etching of Si wafer with DRIE. Channels are fabricated by soft lithography with PDMS. Fluidic access is provided by punching holes on PDMS. PDMS and electrode glass chip are bonded together by curing overnight at $80^{\circ} \mathrm{C}$. The bottom of the glass chip is glued to PCB with epoxy before wire bonding.

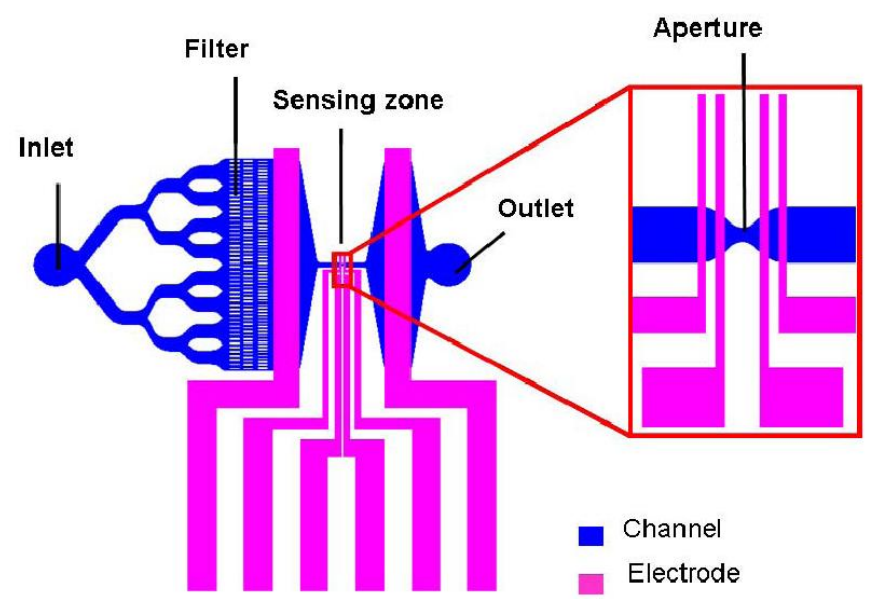

Figure 4: Illustration of the device layout. Particles flow through the filter region before entering the sensing zone. Two large electrodes provide current while the four thin electrodes are used for resistance sensing.

\section{RESULTS AND DISCUSSION}

A fabricated device after assembly and wire bonding to PBS is shown in figure 5 . Testing is performed in a Faraday cage to shield off noise. We tried both DC and low frequency AC as source to pump current into the sensing zone by the external large electrodes. The low frequency $\mathrm{AC}$ is preferred because otherwise DC might irreversibly oxidize the electrodes and introduce diffusion problem for electrolyte to come to the electrode surface.

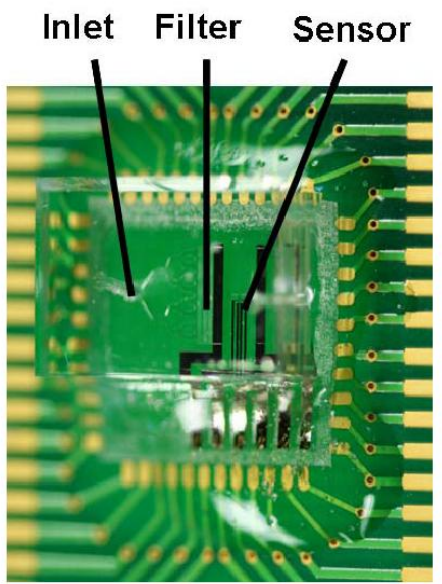

Figure 5. Picture of a fabricated device assembled on PCB.

Fluidic samples are introduced into the device with syringe pump (Pico plus, Harvard Apparatus, MA) with volume flow rate on the order of $0.1 \mu \mathrm{l} / \mathrm{minute}$.

The testing system is shown in figure 6. Low noise amplifier with input resistance larger than $100 \mathrm{G} \Omega$ is used as input buffer stage for sensing electrodes followed by low pass filters to remove high frequency noise. The signals are then amplified by an instrument amplifier before it's converted to DC signal by a true RMS to DC converter. A second stage amplification is used to increase the gain of the overall system before the signal is displayed on an oscilloscope.

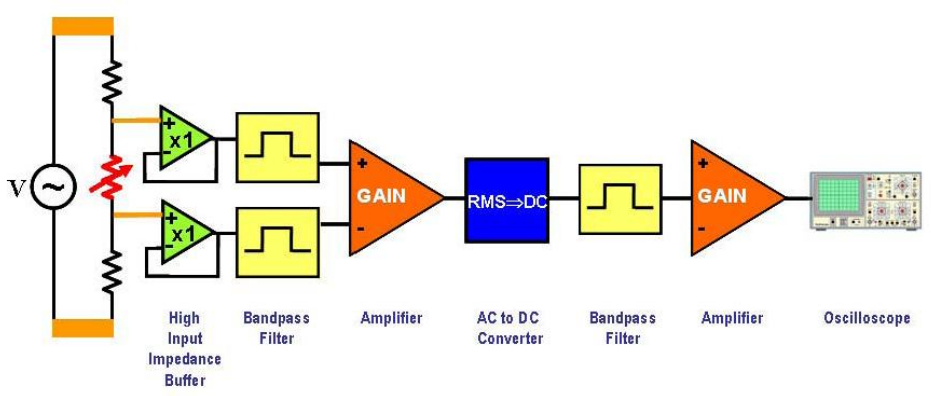

Figure 6. Testing system. A source is connected to the two large electrodes to provide current. Sensing electrodes are first connected to high input impedance buffer amplifier as first stage of signal conditioning.

The operation of the device is confirmed by sensing of polystyrene beads of $5 \mu \mathrm{m}$ and $10 \mu \mathrm{m}$. The beads are dissolved in 65\% Ficoll-Paque Plus (Amersham Biosciences, Sweden) so that the density of the solvent matches the beads. This allows us to perform testing over an hour without sedimentation of the beads. Syringe pump (Pico Plus, Harvard Apparatus, MA) is used to introduce beads into the device.

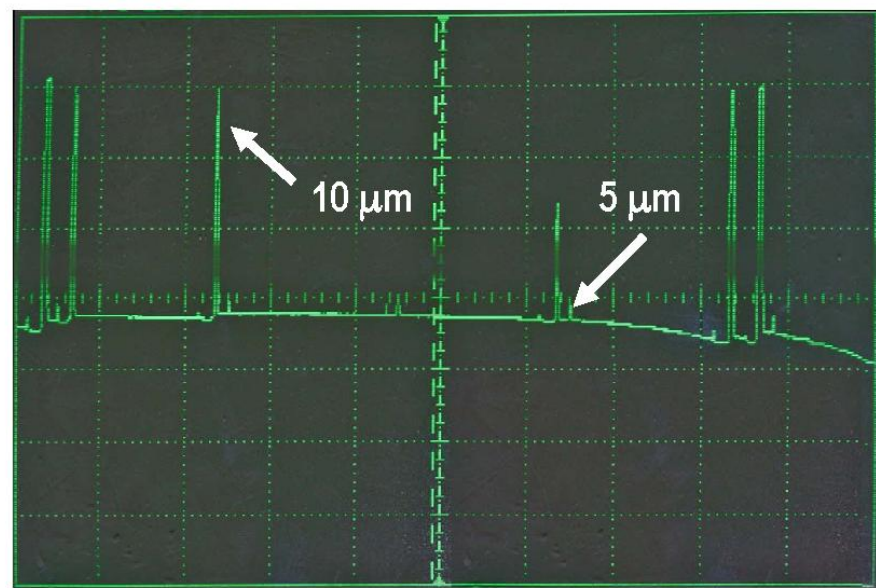

Figure 7. Signals of $5 \mu \mathrm{m}$ and $10 \mu \mathrm{m}$ polystyrene beads measured with oscilloscope. 
Figure 7 shows the result of a mixture of $5 \mu \mathrm{m}$ beads and $10 \mu \mathrm{m}$ beads. The ratio of the height of peaks for $10 \mu \mathrm{m}$ beads to that of $5 \mu \mathrm{m}$ beads matches well with what can be predicted by formula (1). The duration of the pulse matches average flow velocity of the particle. The variation of the height of the signal peak may come from the fact these beads might travel off the central axis of the aperture. Also nonuniformity of the bead size might be responsible.

\section{CONCLUSION AND FUTURE WORK}

We design and fabricated a MEMS devices for impedance sensing of particles at low frequencies. Thin film metal is patterned to be electrodes. To solve the problem of large double layer impedance on electrode electrolyte interface, $\mathrm{Pt}$ black electroplated electrodes with large surface area serve to pump in current, while electrodes with small surface area exposed to electrolyte are used to sense particles near the aperture. A testing system with high input impedance buffer stage is constructed and tested. Experiments with polystyrene beads of different size demonstrate particle sensing with good signal to noise ratio.

Future work will be focused on integration of the current device with sample volume metering for particle counting and improvement of the overall system for handheld application. It can be desirable to design system for high frequency impedance sensing so that the internal structure of the particles can be probed. This will be necessary for applications like leukocyte differential.

\section{ACKNOWLEDEG}

This work is supported by NASA through National Space Biomedical Research Institute (NSBRI). The co-operative agreement number is NCC 9-58-317.

\section{REFERENCES}

[1] H. E. Ayliffe, A. B. Frazier, and R. D. Rabbitt, "Electric impedance spectroscopy using microchannels with integrated metal electrodes," Journal Of Microelectromechanical Systems, vol. 8, pp. 50-57, 1999 .

[2] S. Gawad, L. Schild, and P. Renaud, "Micromachined impedance spectroscopy flow cytometer for cell analysis and particle sizing," Lab on a chip, vol. 1, pp. 76, 2001.

[3] J. H. Nieuwenhuis, F. Kohl, J. Bastemeijer, P. M. Sarro, and M. J. Vellekoop, "Integrated Coulter counter based on 2dimensional liquid aperture control," Sensors and actuators. $B$, Chemical, vol. 102, pp. 44, 2004.

[4] D. W. Lee, S. Yi, and Y.-H. Cho, "A flow-rate independant cell counter using fixed control volume between double electrical sensing zones," presented at 18th IEEE International Conference on Micro Electro Mechanical Systems (MEMS)2005, Miami Beach, Florida, USA, 2005.
[5] D. Satake, H. Ebi, N. Oku, K. Matsuda, H. Takao, M. Ashiki, and M. Ishida, "A sensor for blood cell counter using MEMS technology," Sensors and actuators. B, Chemical, vol. 83, pp. $77,2002$.

[6] H. G. Chun, T. D. Chung, and H. C. Kim, "Cytometry and velocimetry on a microfluidic chip using polyelectrolytic salt bridges," Analytical Chemistry, vol. 77, pp. 2490-2495, 2005

[7] M. P. Maher, J. Pine, J. Wright, and Y. C. Tai, "The neurochip: a new multielectrode device for stimulating and recording from cultured neurons," Journal Of Neuroscience Methods, vol. 87, pp. $45-56,1999$. 